\title{
Contribution à l'étude comparative de la digestion caecale chez les ovins et les caprins : premières observations sur $\mathrm{pH}$, azote ammoniacal et acides gras volatils
}

\author{
C. Masson et F. Faurie
}

ENSSAA, laboratoire de recherches INRA de la chaire de zootechnie, 26 Bd. Docteur-Petitjean, 21000 Dijon, France

(reçu le 29 septembre 1988, accepté łe 26 mai 1989)

Résumé - Cette étude préliminaire de la digestion caecale chez les ovins et les caprins est réalisée sur 8 animaux munis de canules du caecum : 4 boucs castrés et 4 béliers castrés âgés de 2 ans. Deux fourrages sont étudiés successivement : un foin de luzerne-dactyle et une paille d'orge complémentée par du tourteau de soja (120 g par $\mathrm{kg}$ de paille). Le $\mathrm{pH}$ du contenu caecal est en moyenne de 7,8 ; dans l'ensemble, il est plus élevé chez les caprins $(8,0)$ que chez les ovins $(7,7)$ mais la différence n'est statistiquement significative $(P<0,05)$ qu'avec le régime «luzerne". Les teneurs en $\mathrm{NH}_{3}$ et en acides gras volatils totaux sont significativement $(P<0,05)$ plus élevées chez les caprins que chez les ovins. La disparition de la matière sèche in sacco dans le caecum est identique chez les ovins et les caprins quel que soit le temps de séjour dans le caecum (12 h, 24 h, $48 \mathrm{~h})$.

digestion - caecum - ovin - caprin

Summary - A comparative study of caecal digestion in sheep and goats : first observations on $\mathrm{pH}, \mathrm{NH}_{3}$ and volatile fatty acids. This preliminary study of caecal digestion in sheep and goats was carried out on 8 animals with caecum cannulae : 4 castrated he-goats and 4 two-year-old castrated rams. Two forages were studied successively : an orchard-grass-lucerne hay and a barley straw supplemented with soya-bean meal $(120 \mathrm{~g}$ per $\mathrm{kg}$ of straw). The caecal $\mathrm{pH}$ was 7.8 on an average; it was higher in goats (8.0) than in sheep (7.7), but the difference was statistically significant $(\mathrm{P}<0.05)$ only for the "lucerne" diet. The $\mathrm{NH}_{3}$ and VFA contents were higher $(\mathrm{P}<0.05)$ in goats than in sheep. The dry matter disappearance in sacco in the caecum was similar in sheep and goats whatever the transit time in the caecum $(12,24,48 \mathrm{~h})$.

digestion - caecum - sheep - goat

\section{INTRODUCTION}

La digestion caecale chez les petits rumi1969; Bailey \& MacRae, 1970; Hecker, 1971; Orskov et al., 1970) en revanche, aucune étude comparative entre ovins et nants a déjà été abordée (Faichney, caprins n'est signalée. Or, dans notre 
étude comparative de la digestion chez les ovins et les caprins élevés dans les mêmes conditions et alimentés avec des fourrages pauvres (Alrahmoun et al., 1985 et 1986), il est apparu que le temps de séjour dans le compartiment digestif situé après le rumen était plus important chez les caprins que chez les ovins. Aussi avons nous émis l'hypothèse que la digestion caecale chez les caprins leur permettait peut-être une meilleure utilisation des fourrages pauvres, c'est-à-dire que l'activité microbienne caecale des caprins était plus importante que celle des ovins. Dans cette optique, nous avons comparé la digestibilité in sacco dans le caecum d'ovins et de caprins.

\section{MATÉRIEL ET MÉTHODES}

L'expérience porte sur 8 animaux munis de canules du caecum : 4 boucs castrés âgés de 2 ans d'un poids moyen de $43 \mathrm{~kg}$ et 4 béliers castrés également âgés de 2 ans, d'un poids moyen de $44 \mathrm{~kg}$.

Deux régimes de qualité différente sont étudiés successivement pendant 15 jours, après 3 semaines d'accoutumance : un foin de luzernedactyle haché et une paille d'orge hachée complémentée par du tourteau de soja (120 g par $\mathrm{kg}$ de paille).

Les animaux reçoivent 2 repas par jour (à $8 \mathrm{~h}$ et à $16 \mathrm{~h}$ ) et les quantités distribuées d'aliments sont calculées pour couvrir leurs besoins d'entretien. Les canules (diamètre extérieur $3 \mathrm{~cm}$ et longueur $13 \mathrm{~cm}$ ) sont mises en place par le flanc droit de l'animal au milieu du caecum isolé après une incision de $20 \mathrm{~cm}$. Les animaux sont anesthésiés par une injection intramusculaire de Rompum $(0,5 \mathrm{ml})$ puis nous utilisons au cours de l'opération un anesthésiant local, la lurocainne $(5 \mathrm{ml})$.

Le prélèvement de contenu caecal à l'aide d'une pompe à vide s'est révélé délicat, compte tenu de la petite taille du caecum et surtout de la densité du contenu caecal. Nous avons alors effectué des prélèvements passifs basés sur la motricité caecale, 2 fois par jour. Le contenu caecal était récupéré dans un sac attaché hermétiquement à la canule.

Sur les contenus caecaux, nous avons mesuré le $\mathrm{pH}$, dosé l'azote ammoniacal et les acides gras volatils. La digestion in sacco est réalisée avec des sacs de nylon (F100 Tripette et Renaud) de $7 \mathrm{~cm}$ de long et $3 \mathrm{~cm}$ de large. Le fourrage expérimental est broyé à la grille de $0,8 \mathrm{~mm}$ et les sacs sont maintenus dans le caecum pendant $12 \mathrm{~h}, 24 \mathrm{~h}$ et $48 \mathrm{~h}$ seule la disparition de la matière sèche a été étudiée.

\section{RÉSULTATS - DISCUSSION}

Malgré les difficultés rencontrées pour obtenir les prélèvements de contenu caecal, nous pouvons retenir les points suivants (Tableaux I et II).

Le $\mathrm{pH}$ du contenu caecal est en moyenne de 7,8 , il est plus élevé que celui du rumen ce qui confirme les résultats de Faichney (1968) et de Svendsen (1973). II est significativement $(P<0,05)$ plus important chez les caprins $(8,0)$ que chez les ovins $(7,7)$. Toutefois cette différence entre ovins et caprins n'apparaît que pour le régime «luzerne».

Les teneurs en $\mathrm{NH}_{3}$ et en acides gras volatils totaux $\left(\mathrm{AGV}_{\mathrm{T}}\right)$ sont significativement $(P<0,05)$ plus élevées chez les caprins que chez les ovins ce qui pourrait traduire une activité microbienne plus intense dans le caecum des caprins que des ovins. Toutefois, cette différence n'est pas plus importante avec le régime "paille" et l'activité microbienne dans le caecum, quelle que soit l'espèce animale, est plus intense avec le régime "luzerne".

La disparition de la matière sèche in sacco dans le caecum est identique chez les ovins et les caprins et elle est plus élevée avec le régime «luzerne" qu'avec le régime "paille». En moyenne, elle augmente entre $12 \mathrm{~h}$ et $48 \mathrm{~h}$ de séjour dans 
Tableau I. pH, teneurs en ammoniac et en acides gras volatils dans le caecum d'ovins et de caprins.

\begin{tabular}{|c|c|c|c|c|c|c|}
\hline \multirow{2}{*}{$\begin{array}{l}\text { Ration } \\
\text { Paramètres pH }\end{array}$} & \multicolumn{3}{|c|}{ Foin de luzerne-dactyle } & & \multicolumn{2}{|c|}{ Paille d'orge } \\
\hline & $p H$ & $\begin{array}{c}\mathrm{NH}_{3} \\
\mathrm{mg} / 10 \mathrm{ml}\end{array}$ & $\begin{array}{c}A G V T \\
g / l\end{array}$ & $p H$ & \multicolumn{2}{|c|}{$\begin{array}{c}\mathrm{NH}_{3} \\
\mathrm{mg} / 100 \mathrm{~m} / \mathrm{g} /\end{array}$} \\
\hline \multicolumn{7}{|l|}{ Animaux } \\
\hline Ovins & $\begin{array}{r}7,4 \\
+\quad 0,2\end{array}$ & $\begin{array}{r}15,4 \\
\pm \quad 1,8\end{array}$ & $\begin{array}{r}4,4 \\
+1,2\end{array}$ & $\begin{array}{r}8,0 \\
\pm \quad 0,3\end{array}$ & $\begin{array}{r}4,8 \\
\pm \quad 0,9\end{array}$ & $\begin{array}{r}0,9 \\
\pm 0,2\end{array}$ \\
\hline Caprins & $\begin{array}{r}7,9 \\
+0,2\end{array}$ & $\begin{array}{r}25,8 \\
\pm \quad 6,5\end{array}$ & $\begin{array}{r}5,7 \\
\pm 1,8\end{array}$ & $\begin{array}{r}8,1 \\
+\quad 0,3\end{array}$ & $\begin{array}{r}6,5 \\
\pm 1,3\end{array}$ & $\begin{array}{r}1,2 \\
\pm 0,3\end{array}$ \\
\hline
\end{tabular}

Tableau II. Disparition de la matière sèche in sacco après 12,24 et $48 \mathrm{~h}$ dans le caecum d'ovins et de caprins (en \%).

\begin{tabular}{|c|c|c|c|c|c|c|}
\hline \multirow{2}{*}{$\begin{array}{l}\text { Rations } \\
\text { Heures }\end{array}$} & \multicolumn{3}{|c|}{ Foin de luzerne-dactyle } & \multicolumn{3}{|c|}{ Paille d'orge } \\
\hline & $12 h$ & $24 h$ & $48 h$ & $12 h$ & $24 h$ & $48 h$ \\
\hline \multicolumn{7}{|l|}{ Animaux } \\
\hline Ovins & $\begin{array}{r}31,1 \\
\pm 4,3\end{array}$ & $\begin{array}{r}41,5 \\
\pm \quad 7,6\end{array}$ & $\begin{array}{r}54,8 \\
\pm 3,0\end{array}$ & $\begin{array}{r}19,9 \\
+\quad 1,8\end{array}$ & $\begin{array}{r}29,5 \\
\pm \quad 3,5\end{array}$ & $\begin{array}{r}35,6 \\
\pm \quad 3,0\end{array}$ \\
\hline Caprins & $\begin{array}{r}32,3 \\
\pm 5,9\end{array}$ & $\begin{array}{r}44,0 \\
+\quad 4,0\end{array}$ & $\begin{array}{r}55,9 \\
\pm \quad 2,3\end{array}$ & $\begin{array}{r}18,4 \\
\pm 2,2\end{array}$ & $\begin{array}{r}29,4 \\
\pm 2,5\end{array}$ & $\begin{array}{r}35,9 \\
\pm \quad 2,9\end{array}$ \\
\hline
\end{tabular}

le caecum de $74 \%$ et $87 \%$ respectivement pour la «luzerne» et «la paille».

En définitive, ces premières observations laissent à penser que les activités caecales chez les caprins et les ovins sont assez voisines mais d'autres études, avec de nouvelles techniques de prélèvement sont nécessaires pour vérifier ces premiers résultats.

\section{REFERENCES}

Alrahmoun W., Masson C. \& Tisserand J.L. (1985) Etude comparée de l'activité microbienne dans le rumen chez les caprins et les ovins. I. Effet de la nature du régime. Ann. Zootech. 34, 417-428

Alrahmoun W., Masson C. \& Tisserand J.L. (1986) Etude comparée de l'activité microbienne dans le rumen chez les caprins et les ovins. 2. Effet du niveau azoté et de la nature de la source azotée. Ann. Zootech. 35, 109-120 
Bailey R.W. \& Macrae J.C. (1970) The hydrolysis by rumen and caecal microbial enzymes of hemicellulose in plant and digesta particles. $J$. Agric. Sci., Camb. 75, 321-326

Faichney G.J. (1968) Volatile fatty acids in the caecum of sheep. Aust. J. Biol. Sci. 21, 177 180

Faichney G.J. (1969) Production of volatile fatty acids in the sheep caecum. Aust. $J$. Agric. Res. 20, 491-498
Hecker J.F. (1971) Ammonia in the large intestine of herbivores. Brit. J. Nutr. 26, 135-145

Orskov E.R., Fraser C., Mason V.C. \& Man S.O. (1970) Influence of starch digestion in the large intestine of sheep on caecal fermentation, caecal microflora and faecal nitrogen excretion. Br. J. Nutr. 24, 671-682

Svendsen P. (1973) Acetic, propionic and butyric acids in ruminal and caecal digesta of sheep. Nord. Vet. Med. 25, 609-613 